\title{
Le locus mésolithique de Muids « Le Gorgeon-des- Rues » (Eure)
}

A Mesolithic locus at Muids "Le Gorgeon-des-Rues" (Eure)

El locus mesolítico de Muids "Le Gorgeon-des-Rues" (Eure)

\section{Dominique Prost}

\section{(2) OpenEdition}

Journals

Édition électronique

URL : http://journals.openedition.org/rao/2042

DOI : $10.4000 /$ rao.2042

ISBN : 978-2-7535-3432-2

ISSN : 1775-3732

\section{Éditeur}

Presses universitaires de Rennes

\section{Édition imprimée}

Date de publication : 25 décembre 2013

Pagination : $37-55$

ISBN : 978-2-7535-3430-8

ISSN : 0767-709X

\section{Référence électronique}

Dominique Prost, «Le locus mésolithique de Muids « Le Gorgeon-des-Rues » (Eure) », Revue archéologique de l'Ouest [En ligne], 30 | 2013, mis en ligne le 25 décembre 2015, consulté le 05 décembre 2020. URL : http://journals.openedition.org/rao/2042 ; DOI : https://doi.org/10.4000/rao 2042

Ce document a été généré automatiquement le 5 décembre 2020.

Tous droits réservés 


\title{
Le locus mésolithique de Muids « Le Gorgeon-des-Rues » (Eure)
}

\author{
A Mesolithic locus at Muids "Le Gorgeon-des-Rues" (Eure) \\ El locus mesolítico de Muids "Le Gorgeon-des-Rues" (Eure)
}

\section{Dominique Prost}

\section{Présentation}

1 Un diagnostic, mené en 2002 par l'Inrap dans la carrière CSS-Lafarge-Granulat sur la commune de Muids au lieu-dit «Le Gorgeon-des-Rues» (Eure) (Théron et Biard, 2002), avait permis de mettre au jour les traces d'une occupation du Mésolithique localisées dans la partie médiane de la parcelle J71, dans la tranchée TR3. Une première attribution avait été proposée au Mésolithique ancien par M. Biard. Cette découverte inattendue incita le SRA de Haute-Normandie à prescrire une fouille réalisée par l'Inrap sous la direction de l'auteur de cet article en 2003 (Prost et al., 2005). Il s'agit d'une nouvelle découverte mésolithique dont l'intérêt est d'apporter une contribution sur une période encore très peu documentée à l'échelle régionale.

\section{Localisation géographique}

2 La carrière de granulat où fut découvert le site se trouve sur un versant en pente douce $\mathrm{du}$ lobe convexe d'un grand méandre de la Seine entre Gaillon et Louviers sur la commune de Muids (fig. 1). Les communes de Muids et d'Andé se partagent les moitiés est et ouest de ce méandre sur la rive droite du fleuve. Le site préhistorique est implanté à l'extrémité sud-ouest de Muids, en face de l'île de Lormais et des falaises de craie d'Heudebouville situées sur la rive gauche. Il fut repéré au centre de la plaine alluviale inondable dans un espace ouvert où l'altitude du sol naturel des parcelles variait de 10 à 12 mètres avant l'exploitation de carrière et distant de 400 mètres du lit mineur actuel du fleuve. 
Figure 1 : Localisation géographique du site mésolithique à Muids "Le Gorgeon-des-Rues ». Figure 1 : Location of the Mesolithic site at Muids "Le Gorgeon-des-Rues".

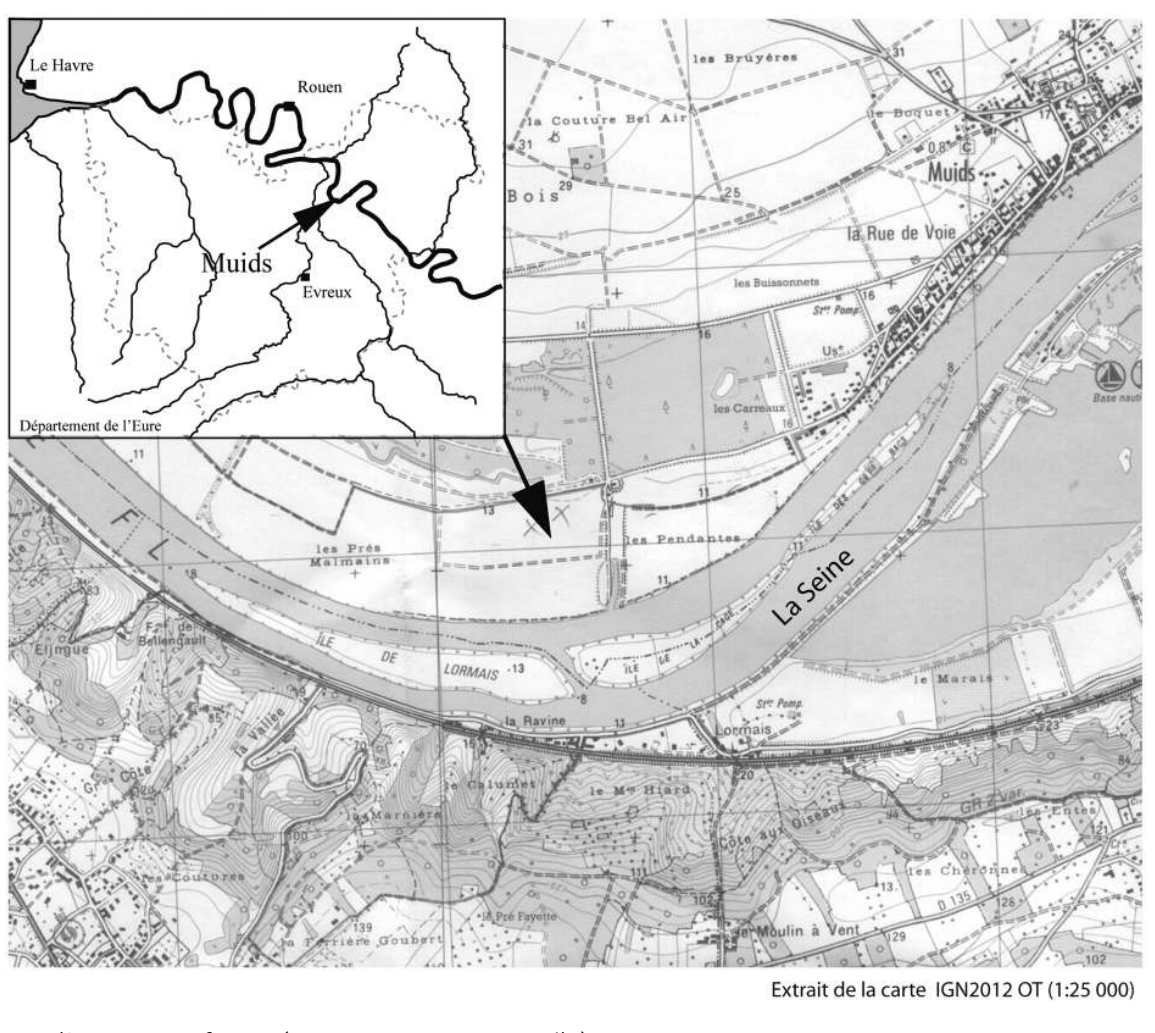

Carrière CSS-Lafarge, (Eure, Haute-Normandie).

\section{Contexte géomorphologique}

Le substrat géologique est une couche épaisse de sables graveleux des alluvions de la Seine dont la partie sommitale qui apparaît à 1,5 mètre de profondeur est datée du Wechsélien. Un long transect (TRA, cf. fig. 2) ouvert perpendiculairement au fleuve a permis de reconnaître les principales couches sédimentaires présentes au-dessus du substrat graveleux (Prost et Biard, 2001). La figure 3 montre une section du relevé de TRA prise à la latitude du locus mésolithique ${ }^{1}$. Dans cette partie du lit majeur de la Seine, une série de six couches de limons de débordement ont recouvert peu à peu la terrasse alluviale. Au cours du Tardiglaciaire, cette plaine était recoupée par des paléochenaux. Le lit de l'un d'entre eux, creusé dans la grave sableuse, a pu être observé dans le transect TRA. Il fut comblé par deux couches alluviales, d'abord par des argiles grises sableuses, marquées par des tâches d'hydromorphie de nappe temporaire $e^{2}$ puis par des limons argileux jaune pâle (US 6). Une première phase de dépôts limoneux de débordement, datée probablement de la fin du Tardiglaciaire, a ensuite recouvert le substrat graveleux et colmaté le paléochenal dont on observe une nette diminution d'épaisseur à mesure que l'on s'éloigne du fleuve. Ce sédiment (US 5) est constitué de limons de teintes hétérogènes brun clair à jaune pâle, carbonatés et à texture compacte, renfermant quelques cailloutis siliceux et calcaires. Une deuxième phase de dépôts de débordement s'est développée avec une épaisseur plus régulière. Cette couche sédimentaire correspond cette fois à un limon argileux fin, brun clair pâle à beige clair, dans lequel apparaît pour la première fois une riche malacofaune. C'est dans cette couche (US 4) que fut localisé le locus mésolithique à 9,69 mètres d'altitude, 
en moyenne. Cette couche, comme la précédente, a épousé le microrelief de cette vallée qui forme, au milieu, une cuvette provoquée par la dépression du paléochenal. Les hommes du Mésolithique se sont installés au bord de cette cuvette à un endroit où les limons de débordement forment par endroits un bourrelet alluvial. Le sédiment de débordement (US 3) qui recouvre partiellement la couche mésolithique est une argile limoneuse compacte, brun foncé à brun jaune, organique, riche en malacofaune et charbons de bois dans laquelle s'est développé un paléosol type pélosol (Duchaufour, 1977). Les charbons de bois, parfois sous forme de fortes concentrations, révèlent la présence de simples foyers à plat ou de souches brûlées liés probablement à des activités de défrichage ou d'écobuage. C'est dans cette couche que furent mises au jour des traces d'occupations du Néolithique moyen et de la protohistoire (Prost et Biard, 2001 ; Prost et al., 2005). Un échantillon de charbons de bois d'une de ces structures de combustion situé à l'interface de US 3 et US 4 dans le secteur A (Prost et al., 2005) fut daté par radiocarbone par le laboratoire de Lyon ${ }^{3}$. L'analyse $14 \mathrm{C}$ a fourni la date $6285 \mathrm{BP}$ (+- 75), soit un âge calibré de 5465 à 5048 av. J.-C.

Figure 2 : Muids « Le Gorgeon-des-Rues » (27).

Figure 2 : Muids “Le Gorgeon-des-Rues" (27).

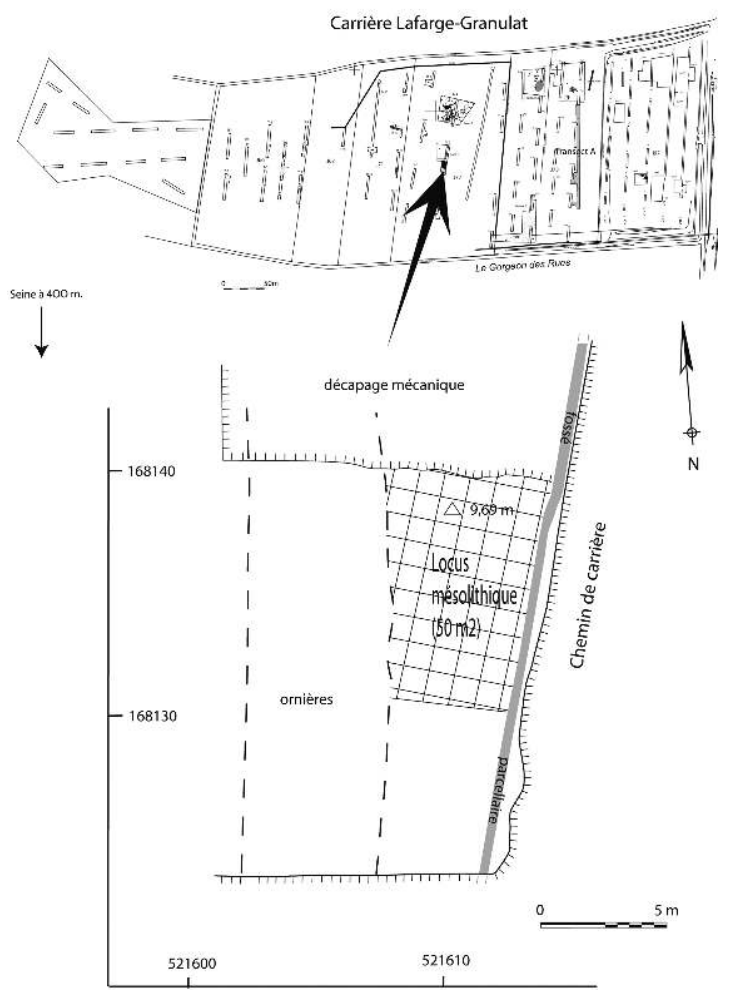

Plan masse des opérations Inrap dans la carrière CSS-Lafarge et du locus mésolithique.

Plan of the excavations carried out by Inrap in the CSS-Lafarge gravel pit and the Mesolithic locus. 
Figure 3 : Muids « Le Gorgeon-des-Rues » (27).

Figure 3 : Muids “Le Gorgeon-des-Rues" (27).

La Seine

Altitude (m)
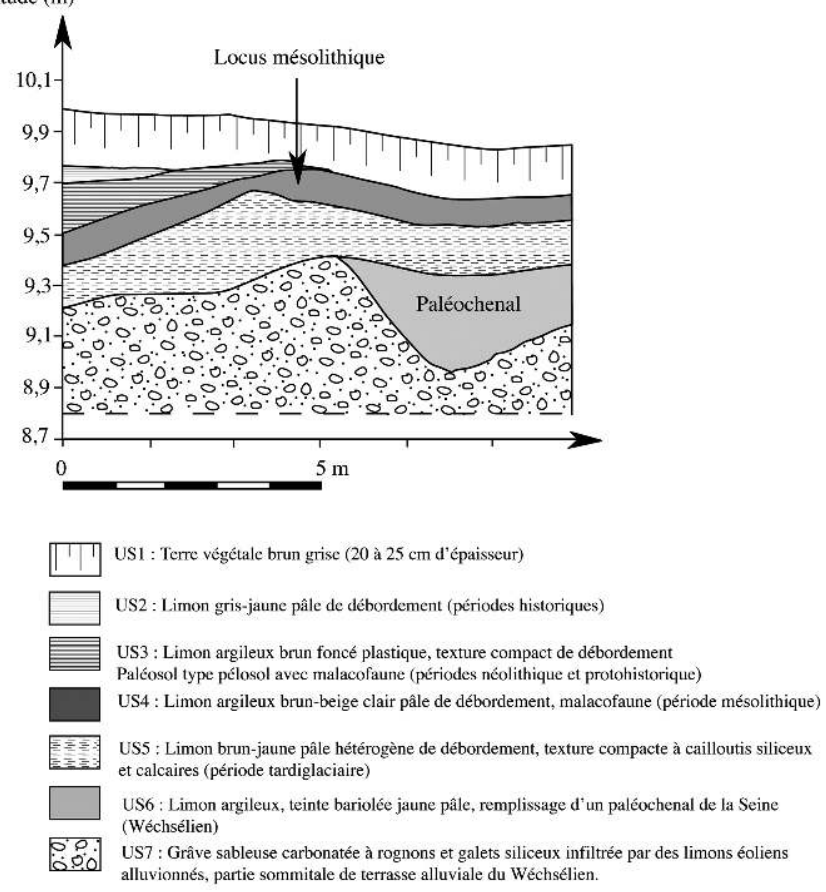

US 3 : Limon argileux brun foncé plastique, texture compact de débordemen Paléosol type pélosol avec malacofaune (périodes néolithique et protohistorique)

US4 : Limon argileux brun-beige clair pâle de débordement, malacofaune (période mésolithique)

US5 : Limon brun-jaune pâle hétérogène de débordement, texture compacte à cailloutis siliceux et calcaires (période tardiglaciaire)

US6 : Limon argileux, teinte bariolée jaune pâle, remplissage d'un paléochenal de ta Seine (Wéchsélien)

US7: Grâve sableuse carbonatée à rognons et galets siliceux infiltrée par des limons éolien alluvionnés, partie sommitale de terrasse alluviale du Wéchsélien.

Contexte et localisation stratigraphique du locus mésolithique.

Context and stratigraphical location of the Mesolithic locus.

\section{État de conservation et mode opératoire de la fouille}

La carrière Lafarge-Granulat fut le théâtre de plusieurs interventions de l'Afan puis de l'Inrap de 1995 à 2004, justifiées par une exploitation progressive de la carrière. La fouille réalisée en 1996 dans la parcelle I 97 (Georges et al., 1996) avait mis en évidence les premières traces d'occupations mésolithiques dans ce secteur. Trois armatures, quelques micro-burins et produits lamellaires furent récoltés au sein d'une nappe de mobilier du Néolithique moyen I dans la partie basse de la plaine alluviale, n'apportant aucune information chronologique précise.

Le locus présenté ici et fouillé en 2003 était beaucoup plus dense et mieux préservé. Néanmoins, lors de la fouille, nous avons constaté qu'une bonne partie du mobilier n'était pas en place. La présence de pièces patinées sur les deux faces, d'autres sans patine, la présence de quelques rares tessons de céramique néolithiques ou protohistoriques au sommet de la couche mésolithique, des silex trouvés de biais ou fichés verticalement montraient clairement que ces vestiges avaient subi de nombreux petits déplacements à maintes reprises. Cela dit, la composition et l'aspect général du mobilier lithique apparaissaient homogènes et cohérents. Situé au beau milieu de la carrière en cours d'exploitation (fig. 4), ce locus fut fouillé totalement sur un peu plus de cinquante mètres carrés (fig. 2). Mais, avant notre intervention, il fut en partie détruit sur son flanc est par l'ouverture d'un chemin de carrière de plus d'1,5 mètre de profondeur, erreur regrettable de topographie que reconnut avoir fait le géomètre de l'entreprise CSS-Lafarge-Granulat. Nous avons repéré également le long du flanc Est un 
ancien fossé de parcellaire traversant la couche mésolithique. Le flanc ouest du locus fut en partie perturbé par le passage d'engins de carrières qui ont creusé des ornières. Les limites d'extension du locus furent toutefois atteintes dans les zones conservées. La fouille fut réalisée manuellement par mètre carré en relevant le mobilier sur plan au $1 / 20^{\mathrm{e}}$. Le sédiment fut systématiquement tamisé sur place.

Figure 4 : Muids « Le Gorgeon-des-Rues » (27).

Figure 4 : Muids "Le Gorgeon-des-Rues" (27).

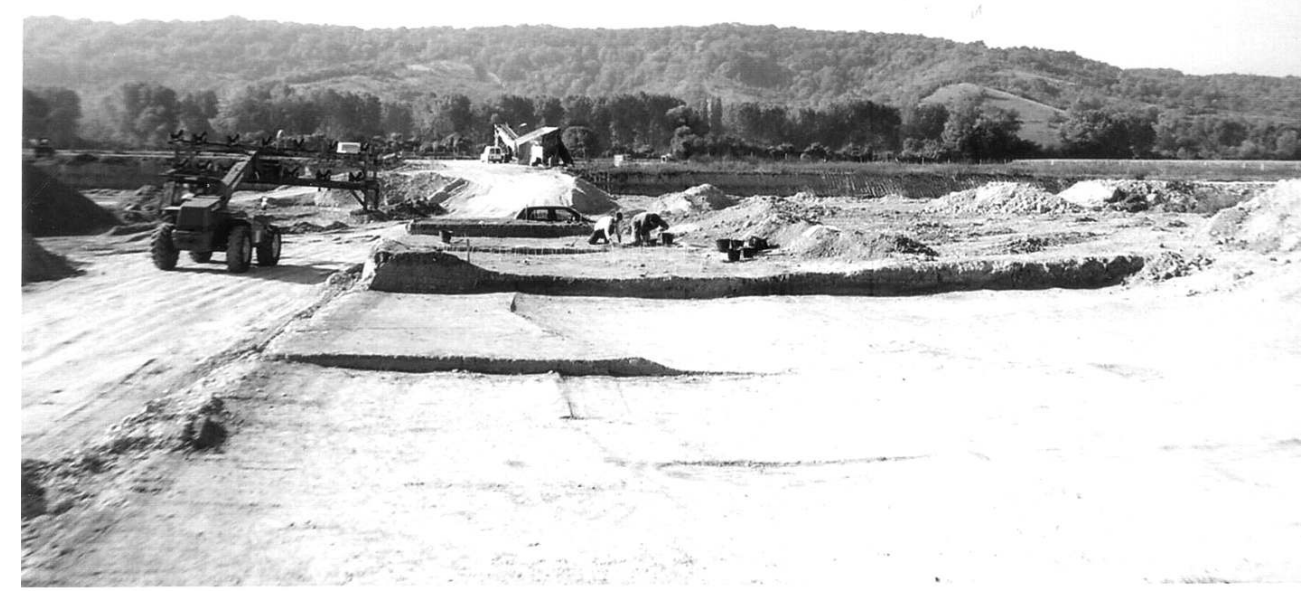

Le locus en cours de fouille au sein de la carrière Lafarge-Granulat en activité, en 2003.

The locus excavation in the Lafarge-Gravel pit in 2003.

Cliché D. Prost.

\section{Étude du mobilier lithique du locus}

63035 pièces ont ainsi été récoltées auxquelles s'ajoutent les 203 pièces recueillies au cours du diagnostic, soit au total 3238 pièces. Cette série est constituée pour une grande part de déchets de taille avec une forte quantité d'esquilles et de petits débris de longueur inférieure à $20 \mathrm{~mm}$ ( 2302 au total). Après sélection, 733 produits ont été conservés pour étude. Sont présents dans cette série 53 outils dont 17 armatures et 21 micro-burins. Notons enfin qu'aucun reste osseux ne fut récolté dans ce locus.

\section{Répartition spatiale}

7 Malgré les problèmes taphonomiques et de conservation rencontrés, nous avons procédé à une analyse spatiale du mobilier par mètre carré. Rappelons que le locus fut en partie détruit avant la fouille. Les limites nord, sud et ouest du locus ont été à peu près circonscrites. Toute la partie est fut emportée par l'ouverture du chemin de carrière. La matrice graphique (fig. 5) montre une répartition hétérogène contrastée, amputée par une partie de la tranchée de diagnostic qui a produit une aire de vide de 
D7 à D10 mais où du mobilier avait été recueilli. Malgré cela, on repère des zones à forte concentration avec plus de trente pièces au mètre carré (exemples en B1 à B4 et en F4, E4, F5, E5) qui sont séparées par des aires de moindre densité, ce qui semble traduire la conservation d'un espace organisé. Cette matrice montre de façon évidente qu'une zone dense devait se poursuivre vers le nord-est. Les pièces de débitage, l'outillage du fonds commun et les armatures suivent grosso modo la même répartition spatiale (fig. 5). En revanche, on a constaté que la répartition des micro-burins était particulière, sans correspondance avec celle des armatures. Quelques silex brûlés ont été récoltés au sein de cette série suggérant la présence d'un foyer à pierres chauffées. Mais en l'absence de trace de rubéfaction et de charbons de bois, il faudrait le situer à l'écart de la zone fouillée.

Figure 5 : Muids « Le Gorgeon-des-Rues » (27).

Figure 5 : Muids "Le Gorgeon-des-Rues" (27).

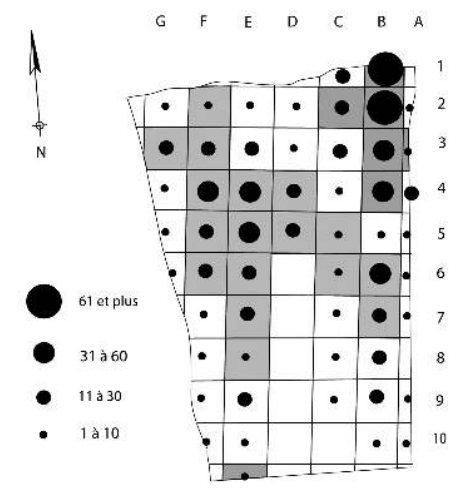

aire de répartition de l'outillage et
des armatures ( 1 a 4 piéces $/ \mathrm{m} 2)$

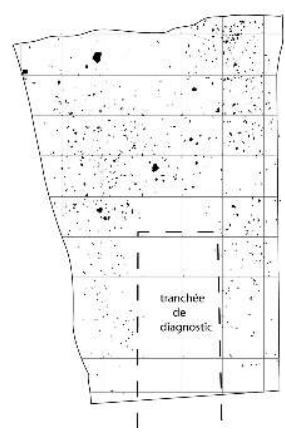

Répartition spatiale quantitative du mobilier par classe au mètre carré du locus mésolithique. Spatial and quantitative distribution of Mesolithic material from locus by $\mathrm{m} 2$.

Des essais de remontage du mobilier ont été tentés en partie pour recueillir des informations sur l'organisation spatiale de ce locus. Les résultats se sont avérés négatifs notamment à cause de la très petite dimension de la majorité des produits et du fait qu'ils étaient très souvent fragmentés. Mais il apparaît évident aussi qu'il manque une partie des pièces qui auraient disparu à l'ouverture du chemin de carrière.

\section{Les matières premières exploitées}

Dans la plaine alluviale du « Gorgeon-de-Rues » couverte de limons de débordement, les blocs de matière première siliceuse sont rares, voire totalement absents. L'étude 
pétrographique des produits montre qu'il s'agit de silex provenant des formations du Crétacé supérieur de la région. Mais sur $60 \%$ d'entre eux, une patine blanc bleutée recouvre la quasi-totalité de leurs faces d'enlèvement, rendant difficile une reconnaissance précise des matières exploitées. Sur les pièces non ou peu patinées, on observe que $81 \%$ d'entre elles, soit une large majorité, sont issues d'un silex brun translucide à cortex blanc crayeux. La structure interne de la matrice est en général bien silicifiée, homogène, sans inclusions majeures. Quelques îlots calcédonieux et des petites géodes de quartz peuvent se trouver dans le cœur du silex. Au sein de ce matériau, on a pu distinguer cinq variantes ou faciès possibles à partir de la nature et l'épaisseur du cortex, de la présence ou non d'un liseré sous-cortical, des teintes et limites avec la matrice. Mis à part ces quelques différences, l'homogénéité de cette matière siliceuse apparaît claire. On a recherché presque exclusivement ce type de silex brun translucide qui devait répondre aux exigences des tailleurs. Ce silex serait d'origine locale. D'après la nature du cortex, Il proviendrait de rognons issus des colluvions de bas de pente ou des vallons encaissés qui s'ouvrent dans les falaises de la Craie.

10 La présence de cinq blocs de ce même silex dont trois testés (pour un poids total de $18,5 \mathrm{~kg}$ ) prouve un acheminement des matières premières sous forme de blocs non débités. Ils ont été recueillis en F2, G2 et en D5, E6, F6, à l'ouest du locus.

\section{Les produits transformés, utilisés}

\section{L'outillage du fonds commun}

11 Les outils retouchés et bruts de débitage identifiés sont au nombre de trente-six, représentant $4,9 \%$ des produits étudiés et $1,1 \%$ de la totalité des produits recueillis dans le locus. Dix-huit outils ont pour support des éclats, six des lamelles, cinq des lames. Les sept autres sont des supports indéterminés, des fragments divers ou sur masse centrale (percuteur). On constate que les lames étaient destinées exclusivement à des outils du fonds commun et non à des supports d'armatures.

Les outils du fonds commun (fig. 6) sont surtout représentés par des pièces a posteriori (pièces brutes utilisées, pièces retouchées partiellement, pièces à coche, pièces à retouches marginales abruptes), c'est-à-dire des pièces ne présentant aucune spécificité typologique particulière. On en compte vingt dont dix sur produits allongés. Les lames ont surtout été utilisées brutes (fig. $6, \mathrm{n}^{\circ} 6$ et 7). Les lamelles peuvent comporter des retouches partielles ou des coches. À moins que ces dernières ne témoignent de fragmentation avortée selon la technique du microburin. 


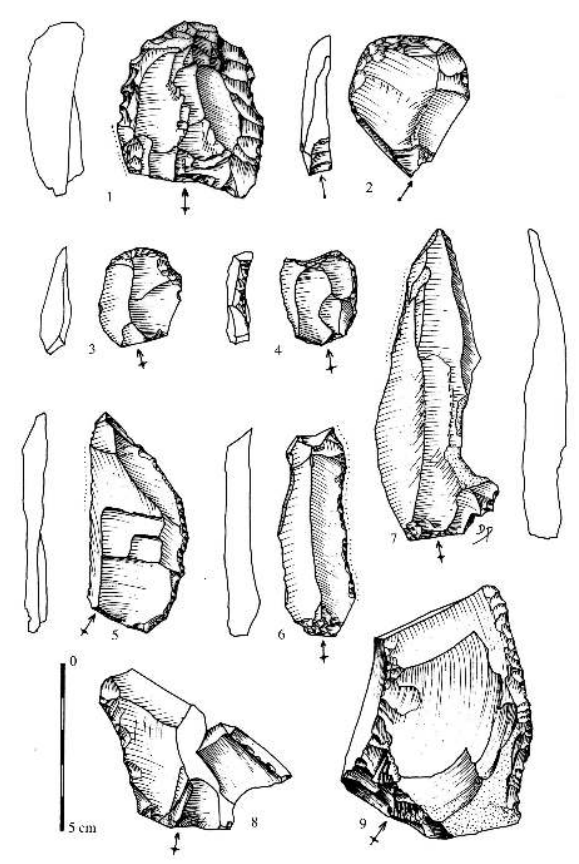

Les outils communs : grattoirs (1 à 4) dont un grattoir denticulé (1); couteau à dos (5) ; lames brutes utilisées (6 et 7); éclat retouché (8) et racloir (9).

The common tools: scrapers (1 to 4) including a denticulate scraper (1); knife (5); used blade (6 and 7); retouched flakes (8).

Sont présents également, par ordre décroissant, cinq grattoirs (fig. 6, $\mathrm{n}^{\circ} 1$ à 4 ) dont quatre sur éclat et un atypique sur lamelle à crête. On notera parmi ces grattoirs un à front denticulé (fig. $6, \mathrm{n}^{\circ} 1$ ). On compte également deux couteaux à dos retouché (fig. 6 , $\mathrm{n}^{\circ} 6$ ), deux pièces mâchurées, un bec, un percuteur, un racloir (fig. $6, \mathrm{n}^{\circ} 9$ ) et une pièce esquillée. Deux pièces comportant plusieurs points d'impact ont été interprétées comme pièces enclume, dont une de grande dimension sur un fragment de galet de $110 \mathrm{~mm}$ de diamètre trouvé au sein d'une des trois fortes concentrations de mobilier en F5.

On notera que les perçoirs et le macro-outillage (pics, outils prismatiques, etc.) sont totalement absents de cette série ainsi que les lamelles Montbani. Les outils percutants sont rares et les burins vrais absents. Seuls, deux grattoirs possèdent à l'extrémité du front un coup de burin à pan très court (fig. $6, \mathrm{n}^{\circ} 2$ ).

Parmi les quatre grattoirs sur éclats, deux d'entre eux peuvent être classés comme grattoirs unguiformes par leurs petites dimensions (fig. 6, $\mathrm{n}^{\circ} 3$ et 4 ).

On constate en général que les supports sont de dimensions très variables et, de ce fait, non standards. À l'inverse des armatures, le choix des supports des outils du fonds commun paraît donc opportuniste. On signalera enfin un grand casson $(90 \mathrm{~mm}$ de long, $65 \mathrm{~mm}$ de large, $25 \mathrm{~mm}$ d'épaisseur) dont la surface convexe, entièrement corticale, comporte plusieurs rainures très fines plus ou moins parallèles probablement produites par un silex. 


\section{Les armatures} par Rozoy, 1967). On compte quatorze pièces entières ou légèrement fragmentées. Cet assemblage est constitué (fig. 7 et tableau 1) de pointes à base retouchée directe et inverse (fig. $7, \mathrm{n}^{\circ} 11$ et 12 ), dont une de forme ogivale retouchée sur un bord latéral, correspondant à une pointe de Tardenois (fig. $7, \mathrm{n}^{\circ} 12$ ). On reconnaît également des triangles isocèles, parfois asymétriques à la limite du scalène (fig. $7, \mathrm{n}^{\circ} 7$ à 9), des segments de cercle dont deux pygmées (fig. $7, \mathrm{n}^{\circ} 13$ à 16 ), deux pointes à troncature oblique dont une pygmée (fig. $7, n^{\circ} 1$ et 3 ) et un fragment de pointe à base oblique non retouchée (fig. $7, n^{\circ} 2$ ). On soulignera également la présence insolite d'une pointe triangulaire à grande troncature oblique et base rectiligne transversale retouchée qui rappelle les pointes de Dreuil (fig. $7, \mathrm{n}^{\circ} 10$ ) et un probable microburin repris comme pointe avec une base retouchée oblique (fig. $7, \mathrm{n}^{\circ}$ ). Les autres pièces fragmentées nous privent malheureusement de données typologiques qui auraient pu enrichir la documentation sur cet assemblage. À noter enfin que les scalènes sont absents ainsi que les trapèzes. Mises à part les pointes fragmentées difficilement identifiables (fig. 7, $\mathrm{n}^{\circ} 2$, 4 et 5) dont l'une d'entre elles pourrait évoquer une pointe de Chaville ${ }^{4}$ (fig. $7, n^{\circ} 2$ ), trois types d'armatures caractérisent cette petite série, à savoir les pointes à base retouchée, les segments et les triangles isocèles. On rappellera parmi elles des pygmées. À noter enfin que les microburins montrent une latérisation à droite nettement majoritaire, seize pièces sur vingt-et-une (tableau 2) et que les armatures retouchées, latérisées à gauche, y sont plus fréquentes (12 sur 17).

Tableau 1 : Décompte des armatures entières par type. Table 1 : List of the complete armatures ordered by type.

\begin{tabular}{|l|l|}
\hline Type d'armatures & quantité \\
\hline pointes à base retouchée & 2 \\
\hline pointes à base oblique non retouchée & 1 \\
\hline pointe de Dreuil (?) & 1 \\
\hline triangles isocèles & 3 \\
\hline segments de cercle & 4 \\
\hline pointes à troncature oblique & 2 \\
\hline pointe indéterminée & 1 \\
\hline total & $\mathbf{1 4}$ \\
\hline
\end{tabular}


Tableau 2 : Latérisation des microburins.

Table 2 : Laterisation of the microburins.

\begin{tabular}{|l|l|l|l|}
\hline Micro-burins & Encoche à gauche & Encoche à droite & total \\
\hline Proximal & 3 & 13 & 16 \\
\hline Distal & 1 & 2 & 3 \\
\hline indéterminé & 0 & 1 & 1 \\
\hline
\end{tabular}

Tableau 3 : Dimensions moyennes des produits (armatures, microburins, lamelles, lames et nucléus).

Table 3 : Average dimensions of the products (armatures, microburins, microblades, blades and core).

\begin{tabular}{|l|l|l|l|l|l|l|l|}
\hline dimensions & $\begin{array}{l}\text { longueur } \\
\text { moy. }\end{array}$ & $\begin{array}{l}\text { écart- } \\
\text { type }\end{array}$ & $\begin{array}{l}\text { largeur } \\
\text { moy. }\end{array}$ & $\begin{array}{l}\text { écart- } \\
\text { type }\end{array}$ & $\begin{array}{l}\text { épaisseur } \\
\text { moy. }\end{array}$ & $\begin{array}{l}\text { écart- } \\
\text { type }\end{array}$ & $\begin{array}{l}\text { rapport } \\
\text { L/l }\end{array}$ \\
\hline armatures & $19,5 \mathrm{~mm}$ & 0,3 & $7,5 \mathrm{~mm}$ & 2,3 & $2,6 \mathrm{~mm}$ & 1,8 & 2,6 \\
\hline microburins & $/$ & $/$ & $10,7 \mathrm{~mm}$ & 3,3 & $2,8 \mathrm{~mm}$ & 3 & $/$ \\
\hline lamelles & $31,4 \mathrm{~mm}$ & 8,8 & $10,2 \mathrm{~mm}$ & 3,3 & $3 \mathrm{~mm}$ & 1,3 & 3 \\
\hline lames & $55 \mathrm{~mm}$ & 12,6 & $22,5 \mathrm{~mm}$ & 4,9 & $7 \mathrm{~mm}$ & 3,2 & 2,4 \\
\hline nucleus & $52 \mathrm{~mm}$ & 11,6 & $/$ & $/$ & $/$ & $/$ & $/$ \\
\hline
\end{tabular}


Figure 7 : Muids « Le Gorgeon-des-Rues » (27).

Figure 7 : Muids “Le Gorgeon-des-Rues" (27).

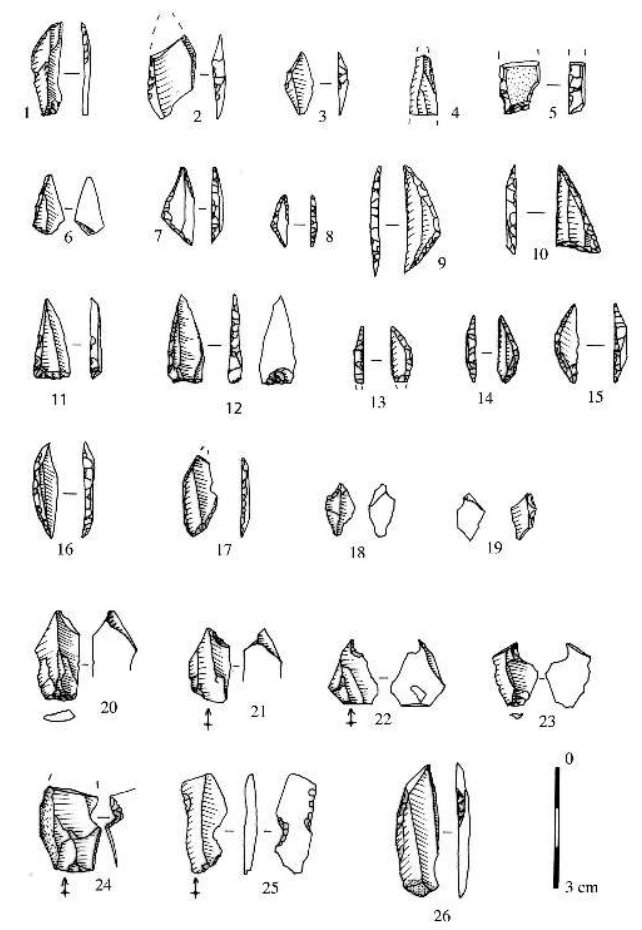

Armatures (1 à 17), microburins (18 à 23), pièces à coche (24 et 25) et piquant trièdre (26) Armatures (1 to 17), microburins (18 to 23), point with notch (24 and 25) and trihedral (26).

\section{Les produits de débitage et leurs caractéristiques techniques}

Sur les 3238 produits récoltés, rappelons que 733 pièces ont été isolées pour l'étude technologique (supports d'outils et armatures compris).

D’après le décompte général (fig. 8), cette série se caractérise par une production de produits allongés (lames et lamelles) qui, ensemble (fragments compris), représente $38 \%$ des produits mais avec une production lamellaire nettement majoritaire par rapport aux lames. Les proportions d'éclats, de lames et de lamelles de plein débitage sont les suivantes : $47 \%$ d'éclats pour $45 \%$ de lamelles et seulement $8 \%$ de lames. Le taux de fragmentation des produits est élevé, principalement pour les lamelles. Il est de $20 \%$ pour les éclats, $50 \%$ pour les lames et $66 \%$ pour les lamelles ${ }^{5}$. 
Figure 8 : Muids "Le Gorgeon-des-Rues » (27). Figure 8 : Muids “Le Gorgeon-des-Rues" (27).

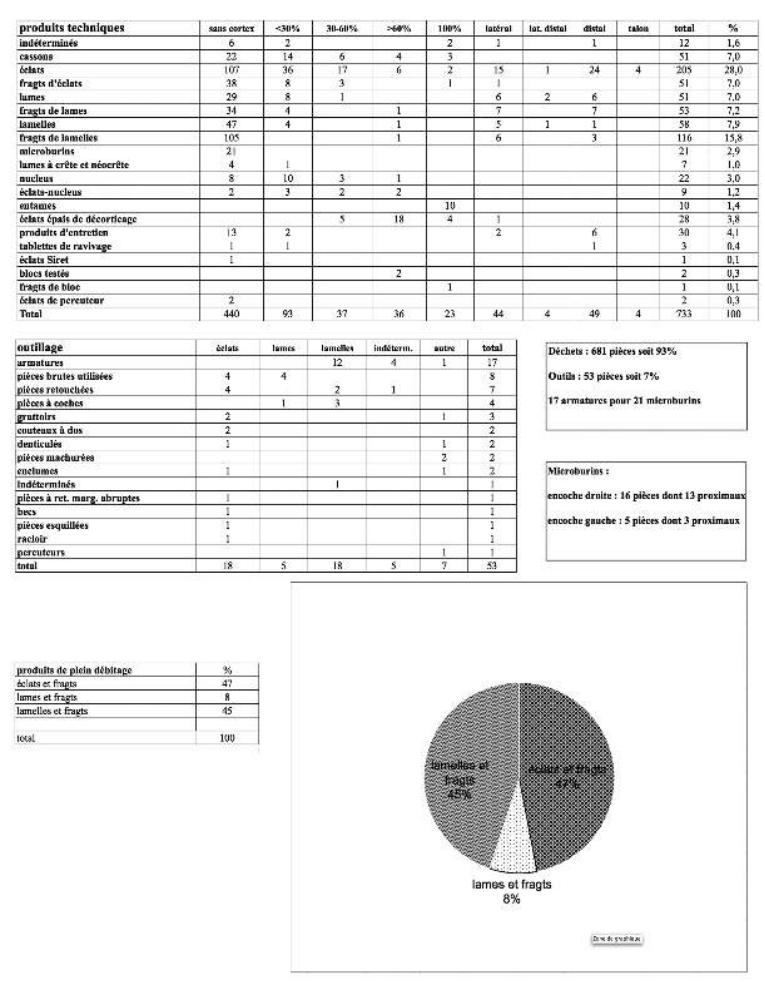

Décompte des produits techniques et de l'outillage (esquilles mises à part) Summary of technical products and tools (except splinters).

\section{Les nucléus}

Trente-et-une pièces ont été récoltées (soit $4,2 \%$ des produits) dont neuf éclat-nucléus. Vingt-et-un nucléus conservent une plage corticale résiduelle, dont trois pièces supérieures à $60 \%$. L'état d'abandon des nucléus est donc assez contrasté, d'autant que certains d'entre eux auraient pu continuer à fournir des enlèvements. De ce fait, l'état d'abandon ne correspond pas toujours à un état d'exhaustion mais à un arrêt de la production voulu par le tailleur.

On reconnaît principalement deux types de nucléus en fonction du mode de débitage : les nucléus à un plan de frappe, les plus nombreux (18 pièces) et les nucléus à deux plans de frappe opposés (sept pièces). Deux autres nucléus ont trois plans de frappe successifs. L'un d'eux montre en fait qu'il y avait, à l'origine, deux plans de frappe opposés. Le tailleur reprit ce nucléus en enlevant sur un des flancs une tablette de ravivage pour poursuivre le débitage sur le plan opposé, l'arête du bord de l'ancien plan de frappe offrant par opportunité une nervure guide. Une des deux lames à crête de cette série est d'ailleurs un bord d'ancien plan de frappe. L'autre nucléus est issu d'un bloc de mauvaise qualité avec plusieurs microgéodes de quartz et les plans de frappe successifs apparaissent en rapport avec la mauvaise qualité du silex.

La morphologie des nucléus à un plan de frappe (fig. 9) est variable et à mettre en relation avec la forme initiale du bloc. L'état de progression du débitage est en général limité. Des nucléus sur éclat ont également été débités selon ce mode opératoire unipolaire. Une plage corticale à l'extrémité distale du nucléus est fréquente. Les flancs 
ont été amincis par le détachement d'éclats larges en vue de resserrer la surface de débitage pour l'obtention de produits allongés. Les plans de frappe sont souvent larges et épais, lisses. La face inférieure des éclats-nucléus a pu être choisie comme simple plan de frappe.

Figure 9 : Muids « Le Gorgeon-des-Rues » (27).

Figure 9 : Muids “Le Gorgeon-des-Rues” (27).
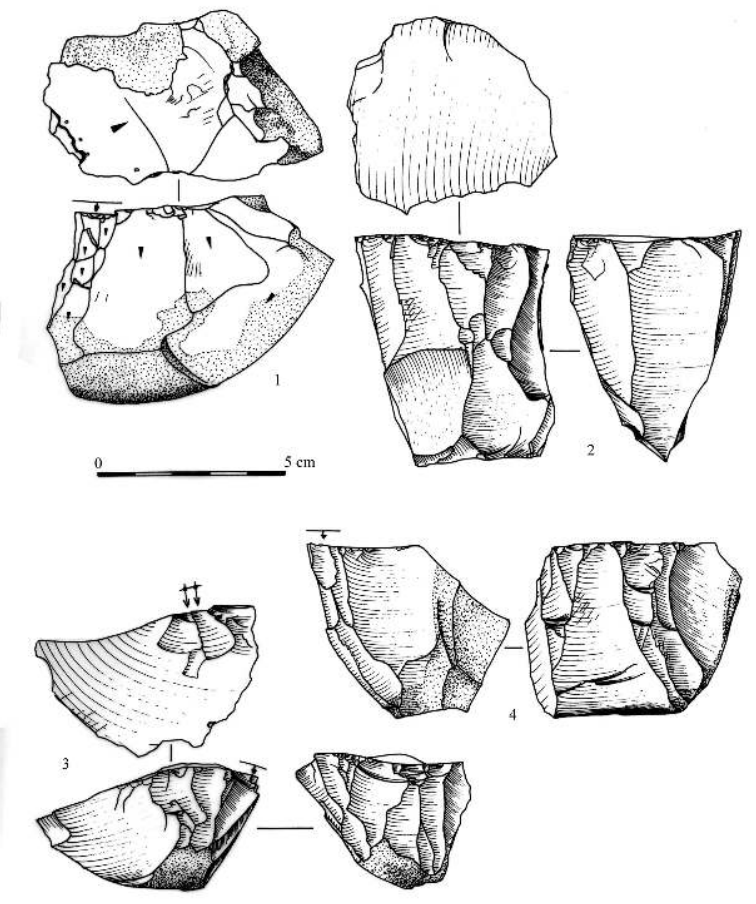

Nucléus à débitage lamellaire et laminaire à un plan de frappe. Core with one striking platform for blade and microblade debitage.

Les nucléus à plans de frappe opposés (fig. 10) montrent, en général, une mise en forme plus élaborée sur tout son pourtour. Les réfections sont fréquentes. Leur forme est, de ce fait, plus régulière. On note des formes coniques, semi-conique, voire en tronc de cône. Deux nucléus sont plus ou moins cylindriques, les deux derniers polyédriques. Tous ces nucléus sont marqués par des plans de frappe horizontaux et/ou inclinés, lisses. Les flancs ont fait l'objet d'un aménagement soigné afin de délimiter une surface de débitage qui est souvent plus étroite que sur les nucléus à plan de frappe unique. 


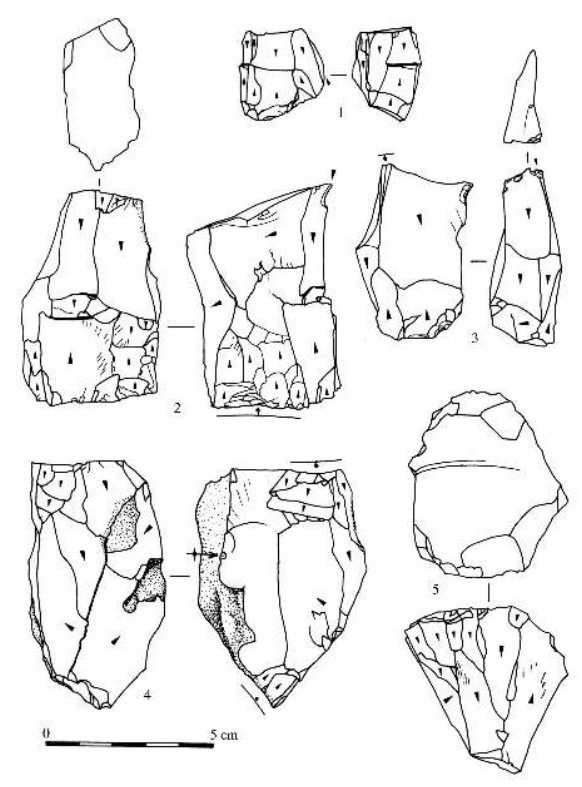

Nucléus à débitage lamellaire et laminaire à deux plans de frappe opposés. Core with two opposite striking platforms for blade and microblade debitage.

La dimension des nucléus, et particulièrement leur longueur (prise dans le sens des derniers enlèvements), est variable. Le plus grand exemplaire a une longueur de $87 \mathrm{~mm}$, le plus petit une longueur de $25 \mathrm{~mm}$, la longueur moyenne enregistrée étant de $52 \mathrm{~mm}$ (tableau 3). L'angle de bord (ou de frappe) moyen, c'est-à-dire l'angle que fait le plan de frappe avec la surface de débitage est de 78 degrés. Il peut être parfois proche de 90 degrés. Il faut noter que la convexité des surfaces de débitage est peu marquée, le plus souvent plate sur les nucléus unipolaires. Elle est plus marquée sur certains nucléus à plans de frappe opposés.

Sur la grande majorité des nucléus, les derniers enlèvements sont la marque d'une production de lamelles ou de petites lames. Les nucléus destinés à une production d'éclats sont absents. Les éclats sont pour l'essentiel des déchets de taille, liés à la mise en forme et à l'entretien des nucléus à lames et à lamelles. Notons enfin que les deux modes de débitage à un ou deux plans de frappe opposés sont présents aussi bien sur les nucléus à lamelles que ceux à lames.

\section{Les produits de décorticage ou d'épannelage}

Ils sont en faible proportion (13\%). Soit ils traduisent un mode opératoire particulier, soit les débuts de la chaîne opératoire s'effectuaient hors de la zone fouillée. 


\section{Les lames et lamelles de plein débitage}

Le débitage était destiné, rappelons-le, essentiellement à une production de lamelles et, dans une moindre mesure, de petites lames à profil rectiligne (fig. 11). La différence entre lames et lamelles tient essentiellement à leur largeur dont la limite à Muids est fixée à $15 \mathrm{~mm}$.

Figure 11 : Muids « Le Gorgeon-des-Rues » (27).

Figure 11 : Muids "Le Gorgeon-des-Rues" (27).
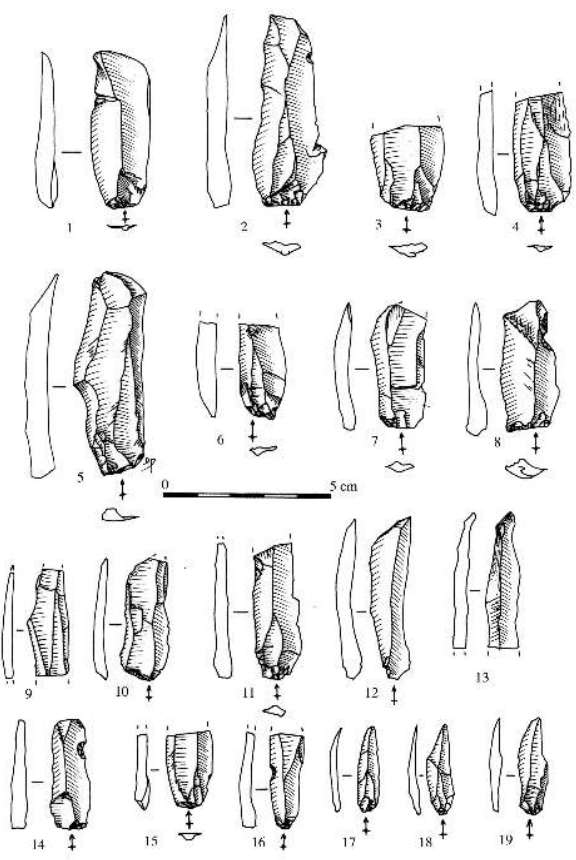

Produits laminaires (1 à 8) et lamellaires (9 à 19) de plein débitage. Blades (1 to 8) and microblades (9 to 19) of full debitage.

Les résultats des analyses morphométriques sur ces produits (tableau 2) montrent que les tailleurs ont produit des lames et lamelles courtes. Bien que les variations extrêmes soient de 18 à $52 \mathrm{~mm}$ pour les lamelles et 40 à $90 \mathrm{~mm}$ pour les lames, la grande majorité de ces produits a une longueur qui n'excède pas $60 \mathrm{~mm}$. La longueur moyenne des lames est de $55 \mathrm{~mm}$, celle des lamelles de $31 \mathrm{~mm}$. Si on réunit ces deux produits, la moyenne est de $43 \mathrm{~mm}$. On notera que la longueur moyenne des nucléus $(52 \mathrm{~mm}$ ) est conforme à celle de ces produits allongés.

Le rapport d'allongement à partir des valeurs moyennes (Longueur/largeur : L/l) donne 2,4 pour les lames et 3 pour les lamelles. Le diagramme de dispersion des longueurs et largeurs (fig. 12) montre que les points s'alignent sur une droite ascendance régulière dont le rapport moyen $\mathrm{L} / \mathrm{l}$ se rapproche de 2,7 . 
Figure 12 : Muids «Le Gorgeon-des-Rues » (27).

Figure 12 : Muids “Le Gorgeon-des-Rues" (27).

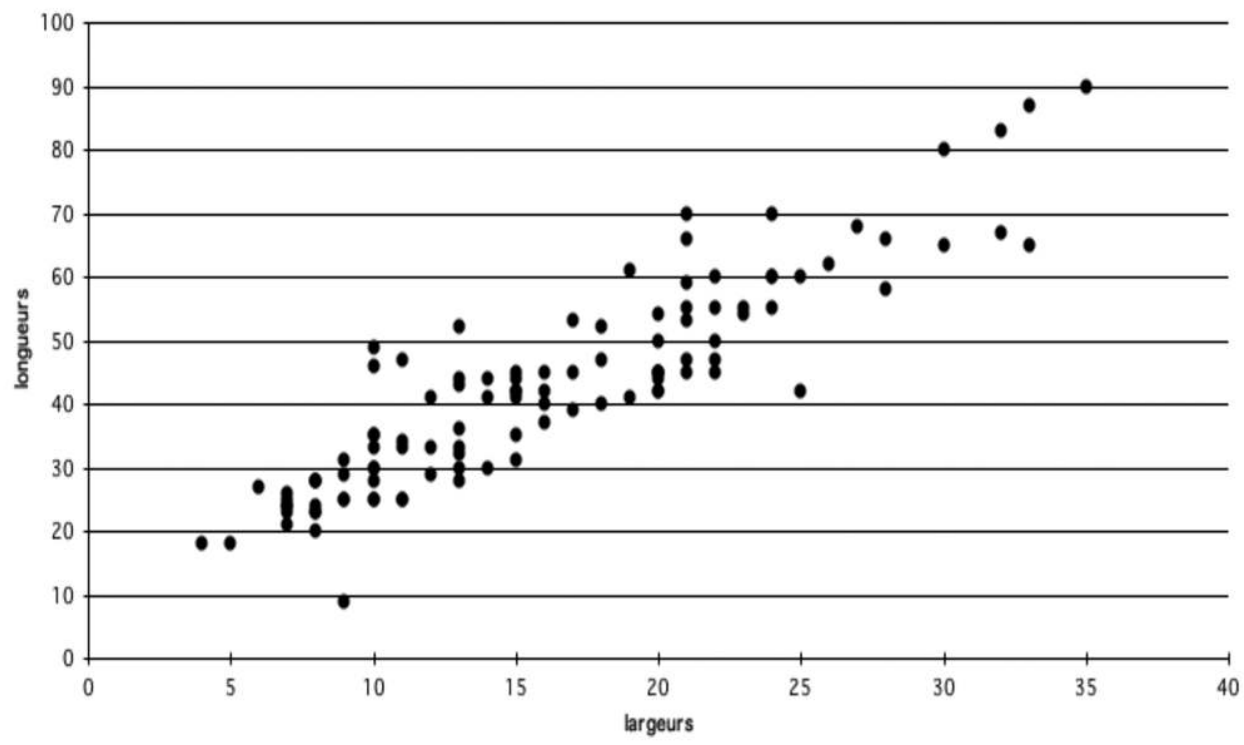

Rapport longueur $(\mathrm{L})$ / largeur $(\mathrm{I})$ des lames et lamelles de plein débitage. Length $(L) /$ with (I) relation of blades and microblades of full debitage lamelles. On constate que le parallélisme des bords latéraux des lames et des lamelles n'est pas régulier (fig. 11). Les profils sont par contre le plus souvent rectilignes comme on l'observe sur les négatifs d'enlèvement des nucléus (fig. 9 et 10). Sur la face supérieure, les négatifs des enlèvements antérieurs laissent des surfaces irrégulières à deux, trois, quatre pans selon les cas, délimités par des nervures non parallèles, sinueuses, de direction variable. Les talons les plus fréquents sont lisses, punctiformes et linéaires, les points d'impact quasiment absents. La corniche est abrasée mais pas systématiquement.

En conclusion, on a affaire à des produits allongés, essentiellement lamellaires, non standardisés, dont le mode de débitage est caractéristique du style de Coincy (Rozoy, 1968).

\section{Les produits d'entretien}

Ils ont pour fonction de raviver le plan de frappe (tablettes de ravivage) et la surface d'enlèvement en préparant ou en corrigeant une nervure guide (lame à crête), en corrigeant le cintrage de la surface d'enlèvement ou encore en supprimant les réfléchissements, les accidents de taille qui bloquent la poursuite du détachement. $\mathrm{Si}$, dans l'ensemble, ces produits sont relativement fréquents (le nombre que nous donnons, trente pièces, soit $4 \%$, est une valeur minimale), on note que les lames à crêtes sont représentées seulement par deux pièces, les néo-crêtes par cinq pièces. La rareté des tablettes de ravivage est à souligner. Tout comme pour les nucléus, on reconnaît deux catégories de produits, les uns issus d'un débitage unipolaire et les 
autres issus d'un débitage bipolaire à deux plans de frappe opposés (fig. 13). Il s'agit de lames et d'éclats ayant emporté tout ou partie de la surface de débitage. Sur les produits d'entretien appartenant à un débitage unipolaire, on observe fréquemment une extrémité distale corticale (fig. $13, \mathrm{n}^{\circ} 1$ à 4 ) dont certaines sont nettement outrepassées (fig. $13, \mathrm{n}^{\circ} 1$ à 3). Les produits d'entretien issus d'un débitage bipolaire comportent, parfois à l'extrémité distale, une partie du plan de frappe opposé (fig. 13, $\left.\mathrm{n}^{05}\right)$.

Figure 13 : Muids « Le Gorgeon-des-Rues » (27).

Figure 13 : Muids "Le Gorgeon-des-Rues" (27).

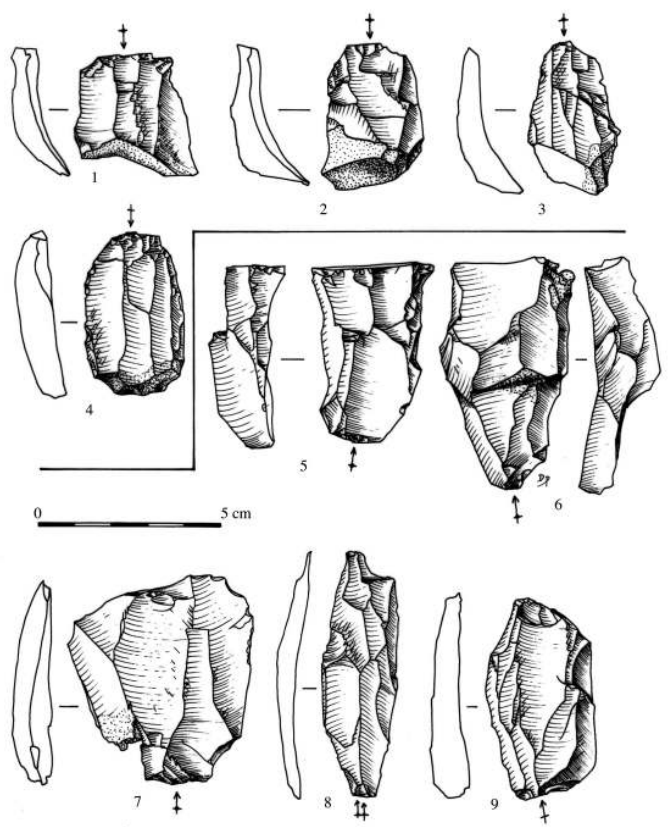

Produits d'entretien issus de débitage unipolaire (1 à 4) et alterné à plans de frappes opposés (5 à 9). Produits from unipolaire debitage ( 1 à 4) and alternating debitage with opposite striking platforms (5 à 9).

\section{Les supports utilisés}

Les supports des armatures étant souvent très transformés, il est difficile de se représenter les formes et les dimensions d'origine. Les armatures ont une largeur moyenne de 7,5 mm avec une variation de 4 à $12 \mathrm{~mm}$ (tableau 3), ce qui laisse supposer que les supports destinés à leur fabrication étaient presque exclusivement des lamelles. Mais les microburins, dont la technique fut largement utilisée pour fragmenter les supports des armatures, fournissent des informations plus précises. Non retouchés, ils ont une largeur moyenne de $10,7 \mathrm{~mm}$ avec une variation de 6 à $19 \mathrm{~mm}$. Ces données sont supérieures à celles enregistrées sur les armatures. Elles relativisent les données sur les supports sélectionnés, parmi lesquels devaient figurer à la fois des lamelles et des lames courtes et petites. Toutefois, parmi ces supports, on ne manquera pas d'indiquer la présence de lamelles pygmées observées pour certaines armatures (fig. 7, 
$\mathrm{n}^{\circ} 8,13$ et 14 ) qui se retrouve également parmi les lamelles non utilisées (fig. $11, \mathrm{n}^{\circ} 17$ à 19).

Mis à part la largeur, l'épaisseur est également un paramètre qui a guidé le choix des supports. On observe une nette distinction entre les supports d'armatures et les microburins d'une part, et les produits de plein débitage non utilisés d'autre part. L'épaisseur moyenne des lames, $7 \mathrm{~mm}$ avec une variation de 2 à $16 \mathrm{~mm}$, et celle des lamelles, $3 \mathrm{~mm}$, avec une variation de 1,5 à $7 \mathrm{~mm}$, contraste avec celle des armatures qui enregistre une valeur moyenne nettement inférieure à $2,6 \mathrm{~mm}$, avec variation de 1 à $3 \mathrm{~mm}$. Les microburins enregistrent une valeur moyenne de 2,8 $\mathrm{mm}$ qui se situent entre celles des armatures et celles des lamelles brutes.

Le profil rectiligne que l'on perçoit sur les supports transformés des armatures ainsi que sur les microburins s'observe aussi sur les produits de plein débitage, tout comme sur la surface de débitage d'une majorité de nucléus et sur le piquant-trièdre (fig. 7, $\mathrm{n}^{\circ} 26$ ). Certains produits d'entretien témoignent aussi de cette rectitude qui semble avoir été un autre paramètre important que les tailleurs ont cherché à contrôler.

\section{Synthèse sur les modalités du débitage lamellaire et laminaire}

\section{Les modalités de préparation et de mise en forme}

D'après les produits présents dans ce locus et les analyses qui ont été faites, il est possible de proposer une reconstitution des premières étapes de la chaîne opératoire du débitage lamellaire et laminaire. La première étape du tailleur semble avoir consisté à fragmenter des blocs de silex (apportés sur le site même) à l'aide de gros percuteurs en pierre dure dans la masse même du silex ou par percussion lancée dont témoigne la présence de gros points d'impact et cônes incipients observables sur différents produits. Ce procédé a pour objectif d'obtenir des cassons, des éclats épais ou des fragments comportant rapidement des surfaces lisses naturelles et angulaires propres à servir de plans de frappe et de surfaces de débitage facilement exploitables, faisant ainsi l'économie d'une phase de décorticage et d'épannelage des volumes. C'est ce qui pourrait expliquer la pauvreté des produits de ce début de chaîne opératoire. Ce mode opératoire est particulièrement adapté à ces blocs de silex, en partie gélifs, fréquents localement, les matières premières brutes apportées sur le site pouvant en témoigner.

L'observation sur plusieurs nucléus d'un plan de frappe lisse, sans trace de préparation, suggère également, à l'origine, l'emploi de ces cassons ou fragments anguleux pour initier le débitage. Par ce procédé, le décalottage est ainsi réduit au minimum.

La phase de mise en forme des nucléus devait donc démarrer rapidement selon un schéma opératoire simple. Elle consistait d'abord à aménager et régulariser les flancs afin de délimiter et resserrer la surface d'enlèvement. Les points d'impact sur les talons des produits de mise en forme (éclats et lames irrégulières plus ou moins épais, souvent corticaux) indiquent l'usage de percuteurs durs par percussion directe au cours de cette phase opératoire. Les lames à crêtes et sous crêtes étant très peu représentées, il semble que cette mise en forme, apte à créer une nervure guide n'était pas toujours nécessaire. La morphologie des cassons anguleux devait fournir, dès le départ, des arêtes naturelles. Il semble également que les petites dimensions des produits recherchés permettent d'expliquer que les tailleurs ont également eu recours à l'usage d'éclats épais comme nucléus. Dans deux cas, des nucléus sur éclat ont été débités sur 
leurs bords latéraux à partir du talon pour l'obtention de lamelles ${ }^{6}$. La face inférieure a pu aussi être utilisée comme plan de frappe, demandant par contre une mise en forme préalable (fig. $9, \mathrm{n}^{\circ} 3$ ).

\section{Les modalités de plein débitage et d'entretien}

L'observation des produits de plein débitage et les analyses morphométriques montrent que l'objectif du tailleur est de contrôler la largeur, l'épaisseur et la rectitude des lamelles pour servir de supports aux armatures. La longueur n'est pas un paramètre de toute première importance, mais la production se limite à des lames et lamelles courtes de moins de $6 \mathrm{~cm}$ de long en général. Le plein débitage s'opère par percussion directe au percuteur tendre. Ce mode de débitage conduit le tailleur à effectuer fréquemment un entretien de la surface de débitage en employant ponctuellement de nouveau le percuteur à la pierre dure aussi bien pour la percussion unipolaire que bipolaire, cette dernière permettant à la fois le détachement de produits d'intention comme celle de supprimer des défauts ou accidents de taille ou de recadrer la rectitude des produits à partir du plan opposé. Le plein débitage semble avoir engendré beaucoup de déchets pour obtenir des supports adéquats comme en témoignent les nombreuses esquilles, petits débris et fragments de lamelles.

\section{Approche chronoculturelle}

41 Sur le plan technique, cette série se caractérise par un débitage de style de Coincy tourné vers une production mixte lamellaire et laminaire dont les lamelles, majoritaires, ont servi de support aux armatures de trait, associée à la technique du microburin. Ce mode de production est commun aux séries des sites du Mésolithique ancien et moyen dans le nord de la France, au sens large. L'outillage du fonds commun, peu caractéristique et en petit nombre, n'apporte pas d'information particulière sur l'appartenance chronoculturelle.

42 C'est sur les armatures que repose l'essentiel de la discussion autour de l'attribution chronologique de ce locus. Nous sommes toutefois confrontés à un assemblage restreint de dix-sept pièces, ce qui limite les possibilités d'interprétation. On ne peut décemment utiliser l'absence de certaines armatures comme un argument pertinent en faveur de telle ou telle hypothèse. On ne peut pas non plus parler de cette composition en termes de fréquence. On peut simplement constater que la superficie de Muids $\left(50 \mathrm{~m}^{2}\right)$ n'est pas tellement plus importante que celle de sites majeurs ayant fourni un nombre élevé d'armatures comme à Acquigny « L'Onglais » (179 armatures sur $\left.69 \mathrm{~m}^{2}\right)$ ou le secteur III des Closeaux à Rueil-Malmaison (101 armatures sur $68 \mathrm{~m}^{2}$; Lang et Sicard, 2008). Sur ce point, le locus de Muids laisse suggérer une occupation ponctuelle ou de courte durée. Ceci dit, les hypothèses que l'on pourrait formuler doivent être prises avec prudence.

L'essentiel de la discussion va porter sur les trois types d'armature qui caractérisent cet assemblage en apparence homogène : les segments, les triangles isocèles et les pointes à base transversale retouchée. Cette composition ternaire est présente sur les sites de la fin du Mésolithique ancien ou du début du Mésolithique moyen dans la moitié nord de la France. Cela dit, il n'y a pas, en Normandie, de sites strictement comparables. On peut d'ores et déjà exclure les sites bas-normands qui appartiennent à d'autres entités chronoculturelles. 

plan technologique, d'après l'étude réalisé par B. Souffi (Souffi, 2003a). Par ailleurs l'auteur montre clairement que ce site forme un palimpseste où se chevauchent deux ensembles distincts. Si des segments et quelques rares triangles isocèles sont présents, l'ensemble A se caractérise surtout par des pointes à troncature oblique et des pointes à dos. Les pointes à base retouchée sont absentes. L'assemblage de l'ensemble $\mathrm{A}$ est à rapprocher des sites de la vallée de la Somme et du secteur IV des Closeaux attribuables à la fin du Préboréal. Par contre, on retrouve, en petit nombre, des segments, des triangles isocèles et des pointes à base retouchée. Mais ces dernières sont de factures différentes de celles de Muids. Cet ensemble fut apparenté au Mésolithique moyen (Boréal). Les assemblages d'Acquigny ne fournissent donc pas suffisamment d'éléments de comparaisons pertinents avec Muids.

Chéronvilliers "Les Essarts» (Paulet-Locard, 1992) qui a fourni trente-et-une armatures offre une comparaison plus intéressante avec ses triangles isocèles fréquents et ses pointes à base retouchée qui sont, comme à Muids, latéralisées à gauche. On y trouve également des pointes à troncature oblique similaires mais également des scalènes. On notera surtout l'absence de segments. Chéronvilliers pourrait présenter un décalage chronologique avec Muids. Mais tous deux semblent montrer des affinités méridionales par la présence d'isocèles qui sont très fréquents sur les sites du Mésolithique ancien, situés entre la Seine et la Loire, néanmoins mal conservés et dont la datation n'est pas assurée. On trouvera, en effet, des similitudes dans l'assemblage des sites d'Autry-le-Chatel (Bazin et al., 1995) ou de Saint-Privé «Les Quatre-Arpents » dans l'Yonne (Huchet et Thévenin, 1995). Mais c'est surtout le site d'Attray dans le Loiret (Girard, 1995) qui présente l'assemblage le plus proche de Muids avec la même composition ternaire des isocèles, segments et pointes à troncature oblique, chacun en quantité similaire. Ce site fut attribué à l'époque par son auteur au Mésolithique ancien (phase ancienne du Beaugencien).

Mais d'un point de vue qualitatif, on ne peut souscrire totalement à une attribution du locus de Muids à un groupe d'influence méridional par la présence d'une pointe de Tardenois dans sa série qui nous oblige à considérer également des affinités nordiques. L'association de pointes à base retouchée (dont la pointe du Tardenois) et de segments nous rapprochent des techno-complexes qu'illustre la culture du Beuronien (Kozlowski, 1983), plus précisément les assemblages à segments du Beuronien nord-occidental (Ducrocq, 2009) où peuvent se retrouver en petite quantité comme à Warluis IIC des pointes à troncature oblique et des pièces mâchurées. Sur la base de plusieurs datations absolues, ces ensembles sont rattachés à la chrono-zone de la première moitié du Boréal (9000-8500 BP), au Mésolithique moyen. Quelques isocèles peuvent compléter ces assemblages mais de façon isolée. Le site de Paris « rue Farman » récemment fouillé (Souffi et al., à paraître) fait apparaître des assemblages beuroniens comparables à ceux du Nord-Picardie. Le locus 2, dont les isocèles sont présents mais rares, est dominé par les pointes à base retouchée et les segments des techno-complexes du Beuronien $\mathrm{A}$ nord-occidental (Souffi et al., à paraître). Par contre, le locus 3 où se retrouvent les pointes à base retouchée dont du Tardenois et les pointes à troncatures obliques, les isocèles y sont plus fréquents alors que les segments sont absents. Ce locus fut attribué 
au début du Boréal, voire à la transition Préboréal-Boréal, conforme aux résultats des datations 14C (Souffi et al., à paraître).

Les isocèles qui sont en général absents ou en position très minoritaire des complexes du nord de la Seine peuvent être interprétés comme à Muids par une influence méridionale du Beuronien A (Séara, 2000) tout comme à Paris « rue Farman ». En effet, il est reconnu que la fréquence des triangles isocèles et l'absence des segments caractérisent en général les territoires du sud de la Seine, notamment entre Seine et Loire $^{7}$. Le locus de Chéronvilliers fut le premier à établir l'hypothèse de possibles influences ou affinités avec le Beuronien A faciès méridional, attribuable à la deuxième moitié du Préboréal pour la Haute-Normandie.

Quant à la pointe de Dreuil (fig. $13, \mathrm{n}^{\circ} 10$ ) à Muids, attribuable aux complexes du Mésolithique final nord occidental, (Ducrocq, 2001), il faut la considérer comme intrusive. Elle n'est toutefois pas totalement isolée sur le site car un trapèze à base retouchée fut récolté durant la fouille du secteur A en amont du locus, à l'intersection des couches US 3 et US 4 (Prost et al., 2005), près du foyer à plat dont la datation 14C pourrait correspondre à cette période.

\section{Conclusion}

Sur la base de quelques sites de référence bien documentés, le locus de Muids pourrait être rattaché dans une fourchette chronologique large de la fin du Mésolithique ancien à la première moitié du Mésolithique moyen. Son appartenance aux techno-complexes du Beuronien nord-occidental des groupes à pointes à base retouchée dont celle du Tardenois et segments est envisageable mais son assemblage, bien que restreint, semble comporter des éléments d'influence méridionale, comme l'illustre le site d'Attray. Le locus de Muids pourrait donc être situé à la chrono-zone de la fin du Préboréal à la première moitié du Boréal. Ce locus confirmerait avec Chéronvilliers que la vallée de la Seine pourrait avoir été, à cette période, une zone tampon entre les groupes nordoccidentaux et méridionaux, dès le Mésolithique ancien. Comme l'avait déjà souligné B. Souffi (Souffi, 2003b), la Seine fournit une frontière naturelle mais aussi une voie navigable essentielle qui se situe au carrefour de territoires de chasse de plusieurs entités culturelles. Ce petit locus à Muids rappelle que la Haute-Normandie comme l'île-de-France s'avèrent des zones géographiques stratégiques dans la recherche sur le Mésolithique ancien et moyen et sur la dynamique de ses populations migratrices.

L'auteur tient à remercier M. Biard, B. Souffi et E. Ghesquière pour leur avis de spécialistes scientifiques sur l'assemblage de ce locus et pour avoir précisé son attribution chronologique ainsi que pour leurs très utiles compléments bibliographiques qu'ils lui ont généreusement adressés. 


\section{BIBLIOGRAPHIE}

BAZIN P., HALLEY J.-P. et THÉVENIN A., 1995 - Les stations mésolithiques d'Autry-le-Chatel (Loiret), Annales littéraires de l'université de Besançon 507, Archéologie 41, p. 181-212, 22 fig. (réf. Girard, 1995).

Duchaufour P., 1977 - « Pédogénèse et classification », in Duchaufour P. et souchier B. (dir.), Pédologie, éd. Masson, 477 p., 92 fig.

Ducroce т., 2001 - Le Mésolithique du bassin de la Somme : insertion dans un cadre morpho-stratigraphique, environnemental et chronoculturel, Publication du CERP 7, Lille.

Ducroce т., 2009 - « Éléments de chronologie absolue du Mésolithique dans le nord de la France », in CROMbé P., VAN STRYDonck M., SERgAnt J., Boudin M. et Bats M. (dir.), Chronology and evolution within the Mesolithic of North-Est Europe, Proceedings of an International Meeting, Brussels, 2007), Newcastle upon Tyne, Cambridge Scholars Publishing, p. 345-362.

GEORGES E., AUBRY B. et ENSENAT D., 1996 - Muids « Le Gorgeon-des-Rues » (Eure), parcelle 197 carrière CSSLafarge, Document final de synthèse de fouille d'évaluation sur extension de carrière, Afan, SRA de Haute-Normandie, 72 p., 36 fig.

GIRARD P., 1995 - «Le gisement mésolithique ancien d'Attray (Loiret) », in Épipaléolithique et Mésolithique du Sénonais et des régions voisines (actes du colloque de Passy, 20-21 novembre 1993), Société archéologique de Sens, Cahier no 2, p. 87-96, 6 fig.

HUCHET A. et THÉVENIN A., 1995 - « Les occupations mésolithiques des Quatre Arpents à Saint-Privé (Yonne) », in Épipaléolithique et Mésolithique du Sénonais et des régions voisines (actes du colloque de Passy, 20-21 novembre 1993), Société archéologique de Sens, Cahier no 2, p. 105-111, 3 fig. коzьowsкі s.-К., 1983 - Cultural differentiation of Europe from 10th to 5th millenium, В.C. Warsaw University Press (réf. Ducrocq, 2009).

LANG L. et SICARD S., 2008 - " Les occupations mésolithiques des Closeaux à Rueil-Malmaison (HauteSeine) ", in Les débuts du Mésolithique en Europe du Nord-Ouest (actes de la table ronde d'Amiens, 9 et 10 octobre 2004), Mémoire de la Société préhistorique française, $n^{\circ} 45$.

PAULET-LOCARD, 1992 - Chéronvilliers, Les Essards, Gallia Information Préhistoire et Histoire no 2, p. 6-9.

PROST D. et BIARD M., 2001 - Muids « Le Gorgeon-des-Rues » site $n^{\circ} 27422$ 007, parcelle J73 carrière CSS-Lafarge, Document final de synthèse de la fouille d'évaluation, Afan, SRA de Haute-Normandie, 26 p., 33 fig.

PROST D., BOULAY G. et THÉRON v. avec la collaboration de GÉHENNE J., 2005 - Muids « Le Gorgeon-des-Rues » parcelle J72 carrière CSS-Lafarge, Rapport final d'opération de fouille, Inrap, SRA de HauteNormandie, 49 p., 52 fig.

Rozoy J-G., 1967 - « Typologie de l'Épipaléolithique franco-belge », Bulletin de la Société préhistorique française, p. 227-260, 2 fig.

Rozoy J.G., 1968 - « L'étude du matériel brut et des microburins dans l'Épipaléolithique (Mésolithique) franco-belge ", Bulletin de la Société préhistorique française, p. 365-390.

SEARA F., 2000 - « Les cadres chronologiques et culturels des occupations mésolithiques de Ruffeysur-Seille « A Daupharde » et de Choisey « Aux Champins » (Jura)», in THÉvenIN A. (dir.), Les derniers 
chasseurs-cueilleurs d'Europe occidentale (13000 - 5500 avant J.-C.), (actes du colloque international de Besançon, 23-25octobre 1998), Presses universitaires franc-comtoises, p. 125-132.

soufri в., 2003a - Le Mésolithique de Haute-Normandie : l'exemple du site d'Acquigny «L'Onglais » (Eure) : contribution à l'étude des gisements mésolithiques de plein-air, Thèse de doctorat de l'université de Paris I Panthéon-Sorbonne, 424 p., 263 fig.

soufri B., 2003b - Le Mésolithique de Haute-Normandie : l'exemple du site d'Acquigny «L'Onglais » (Eure) : contribution à l'étude des gisements mésolithiques de plein-air, Résumé de thèse, Bulletin de la Société préhistorique française, $100\left(\mathrm{n}^{\circ} 4\right)$, p. 807-810.

SOUFFI B., MARTI F., CHAUSSÉ C., GRISELIN S., BRIDAULT A., DAVID E., DRUCKER D.-G., GOSSELIN R., GRANAI S., HAMON C., LEDUX C., VANHAEREN M. et VANTENTIN F. (à paraître) - « Occupations mésolithiques en bord de Seine : le site de Paris « 62 rue H. Farman » $\left(\mathrm{XV}^{\mathrm{e}}\right.$ arrondissement) : organisation et fonctionnement », in VALENTIN B., SOUfFi B., DUCRocQ T., FAgNARD J.-P., SEARA F. et VERJux c. (dir.), Palethnographie du Mésolithique : recherche sur les habitats mésolithiques de plein-air dans la moitié nord de la France et ses marges, (Table ronde de Paris, novembre 2010).

THÉRON v. et BIARD M. avec la collaboration de PRost D., 2002 - Muids « Le Gorgeon-des- Rues » parcelles J67, 71 et 72 carrière CSS-Lafarge, Rapport final d'opération de diagnostic, Inrap, SRA de Haute-Normandie, 6 p., 13 fig.

\section{NOTES}

1. L'abrasion des couches supérieures et l'environnement perturbé n'ont pas permis d'effectuer une coupe stratigraphique complète (couches supérieures tronquées) à l'emplacement du locus mais l'observation d'une coupe à l'extrémité nord du locus a permis de reconnaître la couche mésolithique (US4) identifiée dans le transect TRA à une altitude similaire à la même latitude.

2. Absente de la couche, située dans une partie plus profonde du paléochenal, au nord, dont la largeur du lit dans la coupe de TRA est de 45 mètres.

3. Centre de datation par le radiocarbone, université Claude Bernard Lyon 1, code analyse LY-12863.

4. D'après l'aimable communication d'E. Ghesquière.

5. La limite entre lame et lamelle s'établit au niveau de la largeur des produits fixée à 15 mm pour la série de ce locus.

6. Mode de débitage burinant.

7. Communication d'E. Ghesquière

\section{RÉSUMÉS}

Un locus mésolithique fut fouillé en 2003 sur cinquante mètres carrés dans la carrière CSSLafarge dans la vallée de la Seine à Muids (Eure). La fouille et le précédent diagnostic ont permis 
de récolter 3238 pièces lithiques dont 733 produits techniques étudiables. L'outillage est composé de trente-six outils du fonds commun (grattoirs, couteau à dos, pièce retouchées, etc.) et de dix-sept armatures auxquels s'ajoutent vingt-et-un micro-burins. Les armatures forment un assemblage homogène qui se rattacherait à la fin du Mésolithique ancien ou à la première moitié du Mésolithique moyen, marqué par des influences méridionales.

In 2003, a Mesolithic locus was excavated over an area of $50 \mathrm{~m}^{2}$ in the CSS Lafarge gravel pit in the Seine valley at Muides (Eure). During the excavation 3238 flint artefacts were collected of which 733 technical artefacts were suitable for study. The collection comprises of 53 common tools, 17 armatures and 21 microburins. The armatures make up a very consistent assemblage dating to the end of the Early Mesolithic or the first phase of the Middle Mesolithic, marked by influences from the south.

En 2003, en la cantera CSS-Lafarge en el valle del río Seine en Muids (Eure), se excavó un locus mesolítico sobre una superficie de cincuenta metros cuadrados. Tanto la excavación como el diagnóstico previo ayudaron a recaudar 3.238 piezas líticas de los que 733 productos técnicos se pudieron estudiar. La herramienta se compone de treinta y seis herramientas del fondo común (raspadores, cuchillos, piezas retocadas, etc.) y de diecisiete marcos más veintiún micro cinceles. Los marcos forman un conjunto homogéneo que se relacionaría con el Mesolítico antiguo o la primera mitad del Mesolítico medio, marcado por las influencias del sur.

\section{INDEX}

Mots-clés : Mésolithique, Haute-Normandie, vallée de la Seine, outils en silex, armatures, style de Coincy

Palabras claves : Mesolítico, Alta Normandía, valle del río Seine, herramientas de sílex, marcos, Coincy estilo

Keywords : Mesolithic, Haute-Normandie, Seine valley, flint tools, armatures, Coincy style

\section{AUTEUR}

\section{DOMINIQUE PROST}

Dominique Prost : Ingénieur de recherche Inrap (GO), centre de Recherche du Grand-Quevilly (76) - UMR 6566 Campus de Beaulieu-Rennes I (35) - dominique.prost@inrap.fr . 